\title{
Análise da produção científica sobre plantas com potencial antioxidante, antimicrobiano e alelopático disponíveis na base Scielo
}

\author{
Analysis of the scientific production on plants with antioxidant, \\ antimicrobial and allelopathic potential available in the Scielo base
}

\author{
Aldenir Feitosa dos Santos ${ }^{(1)}$; Jessé Marques da Silva Júnior Pavão( ${ }^{(2)}$; \\ Thiago José Matos Rocha ${ }^{(3)}$; Mayara Andrade de Souza ${ }^{(4)}$; \\ Heloisa Helena Figueredo Alves ${ }^{(5)}$; Helane Carine de Araújo Oliveira ${ }^{(6)}$
}

\footnotetext{
(1)Docente e pesquisadora da Universidade Estadual de Alagoas e membro permanente do Programa de Pós-graduação em Análise de Sistemas Ambientais pelo Centro Universitário Cesmac; Maceió-AL; aldenirfeitosa@gmail.com;

(2) Docente e pesquisador do Programa de Pós-Graduação Análise de Sistemas Ambientais pelo Centro Universitário Cesmac; Maceio-AL; marquesjjunior@gmail.com;

${ }^{(3)}$ Docente e pesquisador da Universidade Estadual de Ciências da Saúde de Alagoas e membro do Programa de Pós-graduação em Análise de Sistemas Ambientais pelo Centro Universitário Cesmac; Maceió-AL; thy_rocha@hotmail.com;

(4)Docente e pesquisadora do Programa de Pós-Graduação Análise de Sistemas Ambientais pelo Centro Universitário Cesmac; Maceio-AL; mayarandrade@hotmail.com;

${ }^{(5)}$ Mestranda do Programa de Pós-Graduação Pesquisa em Saúde, Centro Universitário Cesmac; heloisahelena_alves@hotmail.com; (6) Mestranda do Programa de Pós-Graduação Análise de Sistemas Ambientais pelo Centro Universitário Cesmac; Maceio-AL; heloisahelena_alves@hotmail.com.
}

Todo o conteúdo expresso neste artigo é de inteira responsabilidade dos seus autores.

Recebido em: 25 de agosto de 2018; Aceito em: 30 de agosto de 2018; publicado em 02 de 09 de 2018. Copyright (C Autor, 2018.

RESUMO: O uso de bases de dados bibliográficos tem sido cada vez mais frequente por pesquisadores, permitindo gerar conhecimento atual e determinar novas propostas a partir de informações geradas. Objetivou-se com essa pesquisa realizar uma análise cienciométrica para identificar o número de publicações sobre plantas com potencial antioxidante, antimicrobiano e alelopático, disponíveis nas bases de dados bibliográficos SciELO. Por meio de uma revisão integrativa foram analisadas as variáveis: tipo de expressão de pesquisa utilizada, local de publicação (país), nome da revista, idioma da publicação, tipo de literatura, ano de publicação e área temática da publicação, conforme a organização da plataforma de busca. A partir das bases de dados, foram encontrados 1.013 artigos indexados na Scielo sobre plantas antioxidante, antibacteriana e alelopática, no período de 1983 a julho de 2018, com maior número de publicações no Brasil. Foi detectado um maior número de publicações de plantas antioxidante e antimicrobianas na Revista Brasileira de Farmacognosia, e para plantas alelopáticas destacou-se a revista Planta Daninha. O assunto plantas alelopáticas, independentemente da expressão de pesquisa, foi o que obteve o maior score com média de 93 publicações. O tipo de literatura mais utilizada foi artigo de pesquisa. Foi observado o maior número de publicação nas áreas de ciências agrárias, ciências da saúde e ciências biológicas, para os assuntos plantas antioxidante e antimicrobiana. Já para o assunto plantas alelopáticas destacaram-se as áreas ciências agrárias e ciências biológicas. A pesquisa com plantas antioxidantes e alelopáticas, apresentam um quantitativo maior do que com plantas antimicrobianas, na base Scielo.

PALAVRAS-CHAVE: Bibliographic data. Research platforms. Scielo. Natural products

\begin{abstract}
The use of bibliographic databases has been increasingly frequent by researchers, allowing to generate current knowledge and to determine new proposals from the information generated. The objective of this research was to perform a scientometric analysis to identify the number of publications on medicinal plants with antioxidant, antimicrobial and allelopathic potential, available in SciELO bibliographic databases. By means of an integrative review, the following variables were analyzed: type of research expression used, place of publication (country), name of the journal, language of publication, type of literature, year of publication and thematic area of publication, search platform. From the databases, we found 1,013 articles indexed in Scielo on antioxidant, antibacterial and allelopathic plants, from 1983 to July 2018, with the highest number of publications in Brazil. A greater number of publications of antioxidant and antimicrobial plants were detected in the Brazilian Journal of Pharmacognosy, and the journal Planta Daninha was highlighted for allelopathic plants. The subject allelopathic plants, regardless of the research expression, was the one that obtained the highest score with an average of 93 publications. The most used type of literature was a research paper. The largest number of publications in the areas of agrarian sciences, health sciences and biological sciences was observed for the subjects antioxidant and antimicrobial. On the subject of allelopathic plants, the areas of agrarian sciences and biological sciences were highlighted. The research with antioxidant and allelopathic plants, present a larger quantitative than with antimicrobial plants, in the Scielo base.
\end{abstract}

KEYWORDS: Bibliographic data. Research platforms. Scielo. Natural products. 


\section{INTRODUÇÃO}

Os produtos naturais desempenham um papel importante no tratamento, prevenção de doenças há milhares de anos e continua como fonte de inovação na descoberta de novas drogas. Nesse contexto, as plantas têm uma longa história de utilização no tratamento de doenças humanas e suas utilizações etnofarmacológicas são a fonte primária para a descoberta de novos compostos terapêuticos (JACOB, 2009; MEGRAJ et al., 2011).

A OMS tem expressado a sua posição a respeito da necessidade de valorizar a utilização de plantas medicinais no âmbito sanitário, tendo em conta que 80\% da população mundial utiliza plantas ou preparações delas no que se refere à atenção primária de saúde. Ao lado disso, destaca-se a participação dos países em desenvolvimento nesse processo, já que possuem $67 \%$ das espécies vegetais do mundo (BRASIL, 2013).

O consumo de antioxidantes naturais, como os compostos fenólicos presentes na maioria das plantas que inibem a formação de radicais livres, também chamados de substâncias reativas, tem sido associado a uma menor incidência de doenças relacionadas com o estresse oxidativo (DROGE, 2002). Nos últimos anos, têm-se investigado os efeitos dos antioxidantes em relação às enfermidades, principalmente nos países desenvolvidos do ocidente. As pesquisas têm tentado explicar os benefícios dos antioxidantes nas enfermidades cardiovasculares, em numerosos tipos de câncer, na AIDS, e inclusive em outros diretamente associados com o processo de envelhecimento, como o das cataratas, doença de Alzheimer e outras alterações do sistema nervoso (MORAIS et al, 2009).

A ação antimicrobiana presente nos vegetais das espécies distribuídas nos diferentes Biomas Brasileiros deve-se a biodiversidade e a capacidade de produzirem metabólitos, conhecidas como fintocidas ou substâncias semelhantes a antibióticos. Entretanto a maioria dos vegetais utilizados como fitoterápicos populares não tiveram sua atividade antimicrobiana comprovada. É necessária a realização de estudos que forneçam parâmetros confiáveis que comprovem o real potencial antimicrobiano destes extratos (GONÇALVES; ALVES FILHO; MENEZES, 2005).

As plantas também têm despertado interesse de vários pesquisadores devido ao seu potencial alelopático. A alelopatia é definida como o efeito inibitório ou benéfico, direto ou indireto, de uma planta sobre outra, via produção de compostos químicos que 
são liberados no ambiente. Esse fenômeno ocorre em comunidades naturais de plantas e pode, também, interferir no crescimento das culturas agrícolas (SOUZA et al., 2006), uma vez que as no solo, as substâncias alelopáticas podem combinar-se de várias maneiras e interferir no metabolismo de outros organismos. Muitas substâncias apontadas como alelopáticas estão também relacionadas com funções de proteção ou defesa das plantas contra o ataque de microrganismos e insetos (PIÑA-RODRIGUES; LOPES, 2001). Por esses motivos as plantas alelopáticas tem sido muito pesquisadas como fonte de substâncias inovadoras e eficientes com potencial de uso, em larga escala, na agricultura, tanto para o controle de pragas agrícolas de importância econômica, como, também, no controle de plantas daninhas (SOUZA FILHO et al., 2009).

O Brasil é considerado um dos doze países dotados da chamada megadiversidade, e é signatário da Convenção da Biodiversidade. Nesse contexto, biomas como a Mata Atlântica, é considerada um dos maiores repositórios de biodiversidade do planeta e detém o recorde de plantas lenhosas (angiospermas) por hectare (450 espécies no sul da Bahia), e cerca de 20 mil espécies vegetais, sendo oito mil delas endêmicas, além de recordes de quantidade de espécies e endemismo em vários outros grupos de plantas (VARJABEDIAN, 2010).

O Cerrado é o segundo maior bioma brasileiro, sendo superado em área apenas pela Amazônia. Ocupa 21\% do território nacional, a biodiversidade do Cerrado é elevada, porém geralmente menosprezada. O número de plantas vasculares é superior àquele encontrado na maioria das regiões do mundo: plantas herbáceas, arbustivas, arbóreas e cipós somam mais de 7.000 espécies (MENDONÇA et al., 1998).

Técnicas para quantificar dados etnobotânicos têm sido empregadas como complementares aos levantamentos sobre o uso de plantas por populações. Existem vários métodos quantitativos voltados para avaliar o conhecimento científico, dentre os quais vale destacar a cienciometria (VENDRUSCOLO; MENTZ, 2006).

O objetivo do presente trabalho foi realizar uma análise cienciométrica para identificar o número de publicações sobre plantas com potencial antioxidante, antimicrobiano e alelopático, disponíveis na base de dados bibliográficos SciELO. 


\section{PROCEDIMENTO METODOLÓGICO}

Trata-se de uma revisão integrativa da literatura que tem sido apontada como uma ferramenta que reúne e sintetiza os estudos realizados sobre um determinado assunto, permitindo gerar uma fonte de conhecimento atual sobre o problema e determinar se o conhecimento científico é válido para ser utilizado na prática.

As etapas adaptadas para o desenvolvimento da revisão integrativa foram: levantamento da questão da pesquisa ou definição do problema a ser pesquisado, categorização dos estudos com a síntese dos principais achados e análise detalhada, interpretação dos resultados com a identificação de conclusões e implicações resultantes da revisão integrativa.

Foi selecionada como bases de dados bibliográficos a SciELO - Scientific Electronic Library Online por ser uma biblioteca eletrônica que abrange uma coleção selecionada de periódicos científicos brasileiros. É a maior provedora de periódicos indexados pelo Diretório de Periódicos de Acesso Aberto (Directory of Open Access Journals - DOAJ). Além disso, provém a maioria dos periódicos latino-americanos, de acesso aberto, indexados pela Web of Science e Scopus (PACKER; MENEGHINI, 2014). Não foram adotados critérios de inclusão ou exclusão uma vez que foram utilizados todas as categorias presentes na base de dados bibliográficos SciELO. Para a busca dos descritores padronizados, lançou-se mão do MeSH (Medical Subjects Headings) e o DeCS (Descritores em Ciências de Saúde), utilizando-se: plant / planta, antioxidants / antioxidantes, anti-bacterial agents / antibacterianos e allelopathy / alelopatia.

As variáveis escolhidas para delimitar e nortear o trabalho foram: tipo de expressão de pesquisa utilizada, local de publicação (País), nome da revista, idioma da publicação, tipo de literatura, ano e área temática da publicação, conforme a organização da plataforma de busca. Em seguida, os dados foram tabulados e organizados em uma planilha de acordo com cada variável da pesquisa, conforme já mencionado. Posteriormente, os dados foram analisados por meio de estatística descritiva utilizando programa Bioestat 5.3. 


\section{RESULTADOS E DISCUSSÃO}

Para a análise cienciométrica sobre plantas medicinais com atividade antioxidante, antibacteriana e alelopática, foram obtidos 1.013 artigos indexados no banco de dados SciELO, publicados no período de 1983 (ano em que ocorreu a primeira publicação sobre o assunto) a julho de 2018, utilizando as expressões de pesquisa utilizando os descritores nos idiomas português e inglês (Tabela 1). Pôde ser observado que ocorreu uma variação no quantitativo de publicações recuperadas em função da expressão de pesquisa utilizada para cada assunto, o que caracteriza a importância da elaboração de uma "boa" expressão de pesquisa, para que se possa obter os resultados esperados e evitar os falsos negativos. Pode-se dizer, então, que, para se obter determinada resposta, é preciso fazer a pergunta correspondente. Mas para fazer "boas perguntas", ou perguntas pertinentes e de interesse, é preciso conhecer com antecipação as possíveis respostas, que consistiriam no "Universo de Respostas" (TEMPORINI, 1995). Ou seja, é necessário conhecer as características da base de dados bibliográficos em que se estar navegando, para que se possa elaborar uma expressão de pesquisa adequada.

Foram registradas muitas publicações para os assuntos plantas antioxidantes e plantas alelopáticas, independente da expressão de pesquisa utilizada. Entretanto, para o assunto plantas antimicrobianas, as expressões de pesquisa utilizadas não promoveram o resgate de um número significativo de publicações. O quantitativo de publicação foi de apenas 26 documentos, o que representa pouco mais de $15 \%$, quando comparado com os resultados obtidos para os ouros dois assuntos pesquisados neste trabalho. Este dado nos leva a considerar que talvez, para esse assunto, essa não seja e expressão de pesquisa adequada para a base de dados bibliográficos SciELO (Tabela 1). 
Tabela 1. Expressões de pesquisa utilizadas na busca na base de dados bibliográficos

SciELO

\begin{tabular}{|c|c|c|c|}
\hline ASSUNTO & EXPRESSÃO DE PESQUISA & SIGLA & No PUBLICAÇÕES \\
\hline \multirow{2}{*}{$\begin{array}{l}\text { PLANTAS } \\
\text { ANTIOXIDANTES }\end{array}$} & $\begin{array}{c}\text { plant antioxidant AND type:("research- } \\
\text { article" OR "review-article") }\end{array}$ & EP1 & 558 \\
\hline & $\begin{array}{c}\text { planta antioxidante AND type:("research- } \\
\text { article" OR "review-article") }\end{array}$ & $\mathrm{EP} 2$ & 166 \\
\hline \multirow{2}{*}{$\begin{array}{l}\text { PLANTAS } \\
\text { ANTIMICROBIANAS }\end{array}$} & $\begin{array}{c}\text { (plant) AND (anti-bacterial) AND } \\
\text { type:("research-article" OR "review-article") }\end{array}$ & EP1 & 21 \\
\hline & (planta) AND (antibacterianos) & $\mathrm{EP} 2$ & 5 \\
\hline \multirow{2}{*}{$\begin{array}{c}\text { PLANTAS } \\
\text { ALELOPÁTICA }\end{array}$} & $\begin{array}{c}\text { (planta) AND (alelopatia) AND } \\
\text { type:("research-article" OR "review-article") }\end{array}$ & $\mathrm{EP} 1$ & 134 \\
\hline & $\begin{array}{c}\text { (plant) AND (allelopathic) AND } \\
\text { type:("research-article" OR "review-article") }\end{array}$ & $\mathrm{EP} 2$ & 129 \\
\hline \multicolumn{3}{|c|}{ TOTAL DE PUBLICAÇÕES } & 1.013 \\
\hline
\end{tabular}

Com relação aos países que apresentam o maior número de publicações sobre os assuntos pesquisados, destacou-se independente da expressão de pesquisa utilizada, o Brasil. Isso pode ser explicado pelo fato de que o funcionamento regular do SciELO teve início no Brasil em 1998, depois de um projeto-piloto de um ano em parceria com a Fundação de Amparo à Pesquisa do Estado de São Paulo (FAPESP) e o Centro LatinoAmericano e do Caribe de Informação em Ciências da Saúde (BIREME/OPAS/OMS). O SciELO foi concebido como um projeto e uma estratégia para superar o fenômeno conhecido como "ciência perdida", causado pela presença muito fraca dos periódicos de países em desenvolvimento nos índices internacionais. Além da falta de visibilidade, este fenômeno também se manifestou na falta de comunicação, em muitas áreas, entre os pesquisadores de países desenvolvidos e em desenvolvimento, bem como entre os pesquisadores de países em desenvolvimento (PACKER; MENEGHINI, 2014). 
Figura 1. Relação de países que publicam os assuntos pesquisados na base de dados bibliográficos SciELO
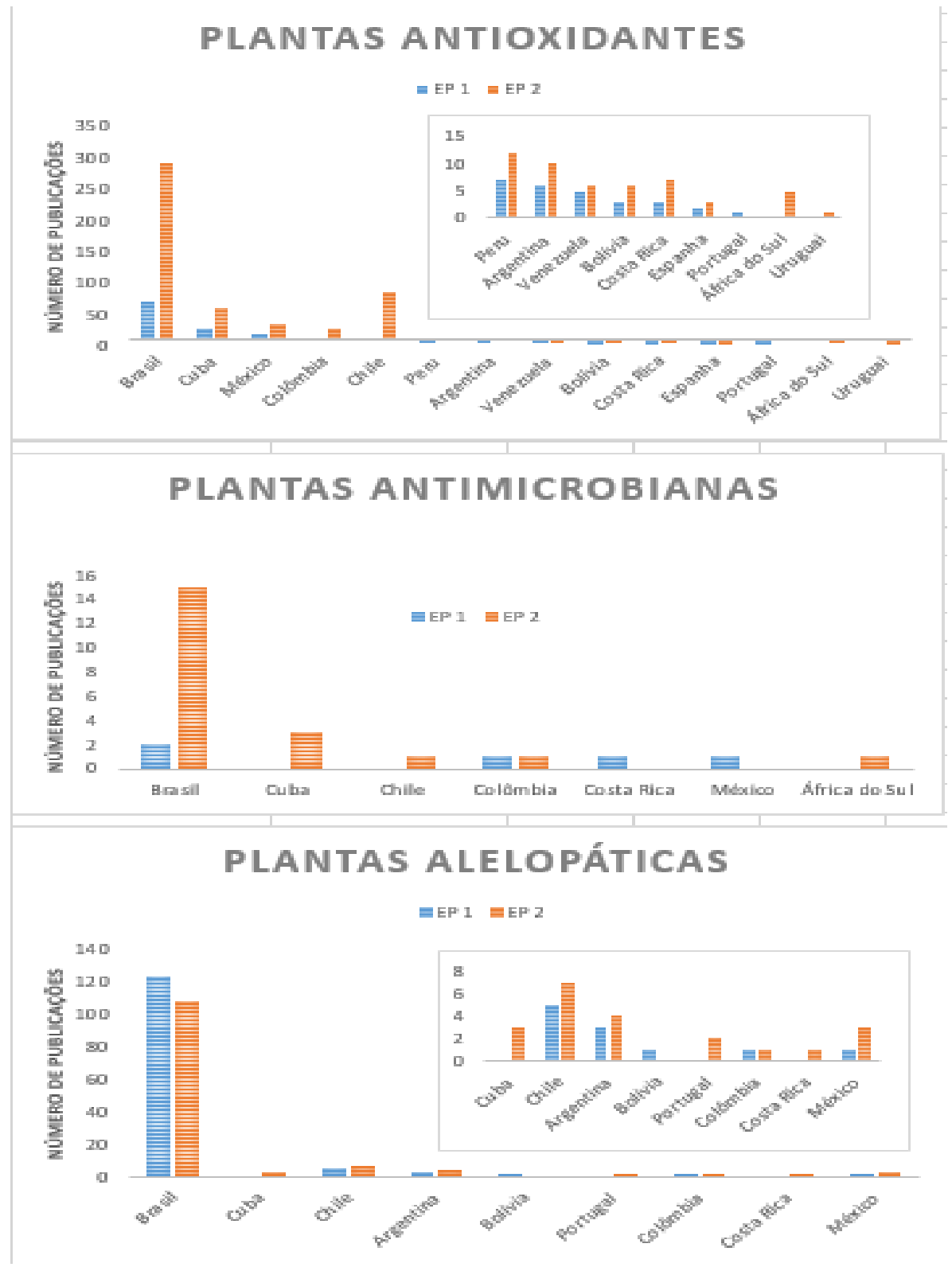

EP 1- expressão de pesquisa utilizando descritores em português; EPQ expressão de pesquisa utilizando descritores em inglês. $\mathrm{O}$ detalhamento das expressões consta na tabela 1.

As revistas que mais publicaram artigos sobre atividade antioxidante em plantas, quando se usou a expressão em Inglês (plant AND antioxidant), foram Revista Brasileira de Farmacognosia (52 publicações), Revista Cubana de Plantas Medicinales (44 publicações) e Journal of soil science and plant nutrition (37 publicações). Ainda sobre a atividade antioxidante em plantas, quando utilizou-se a expressão de pesquisa em 
Português (EP2), os periódicos que mais publicaram foram Revista Cubana de Plantas

Medicinales (19 publicações), Revista Brasileira de Farmacognosia (15 publicações) e Revista Brasileira de Plantas Medicinais (13). Quando utilizadas as expressões de pesquisa (plantas AND alelopatia e plant AND allelopathic) houve um certo equilíbrio quanto ao número de publicações, o periódico que mais apresentou publicações para as expressões utilizadas foram, Planta Daninha com 93 publicações quando se utilizou EP1 e 42 publicações quando se utilizou EPQ (Tabela 2 ).

Tabela 2. Análise de periódicos publicados nacionais e internacionais em função das Expressões de Pesquisa utilizadas

\begin{tabular}{|c|c|c|c|c|c|c|}
\hline $\begin{array}{l}\text { Expressão } \\
\text { de pesquisa }\end{array}$ & Planta Antioxidante & $\mathbf{N}$ & $\begin{array}{c}\text { Planta } \\
\text { Antibacteriana }\end{array}$ & $\mathbf{N}$ & $\begin{array}{c}\text { Planta } \\
\text { Alelopática }\end{array}$ & $\mathbf{N}$ \\
\hline \multirow{7}{*}{$\mathrm{EP} 1$} & $\begin{array}{l}\text { Revista Cubana de } \\
\text { Plantas Medicinales }\end{array}$ & 19 & $\begin{array}{c}\text { Revista Brasileira de } \\
\text { Farmacognosia }\end{array}$ & 2 & Planta Daninha & 93 \\
\hline & $\begin{array}{c}\text { Revista Brasileira de } \\
\text { Farmacognosia }\end{array}$ & 15 & $\begin{array}{l}\text { Acta Biológica } \\
\text { Colombiana }\end{array}$ & 1 & $\begin{array}{c}\text { Acta Botanica } \\
\text { Brasilica }\end{array}$ & 4 \\
\hline & $\begin{array}{l}\text { Revista Brasileira de } \\
\text { Plantas Medicinais }\end{array}$ & 13 & Outras & 2 & $\begin{array}{c}\text { Revista Brasileira } \\
\text { de Plantas } \\
\text { Medicinais }\end{array}$ & 3 \\
\hline & $\begin{array}{l}\text { Anais da Academia } \\
\text { Brasileira de Ciências }\end{array}$ & 6 & & & $\begin{array}{l}\text { Chilean journal of } \\
\text { agricultural } \\
\text { research }\end{array}$ & 2 \\
\hline & Planta Daninha & 5 & & & Acta Amazonica & 1 \\
\hline & $\begin{array}{l}\text { Brazilian Journal of } \\
\text { Pharmaceutical } \\
\text { Sciences }\end{array}$ & 4 & & & Outras & 31 \\
\hline & Outras & 86 & & & & \\
\hline \multirow{7}{*}{$\mathrm{EP} 2$} & $\begin{array}{c}\text { Revista Brasileira de } \\
\text { Farmacognosia }\end{array}$ & 52 & $\begin{array}{l}\text { Revista Brasileira de } \\
\text { Farmacognosia }\end{array}$ & 3 & Planta Daninha & 42 \\
\hline & $\begin{array}{l}\text { Revista Cubana de } \\
\text { Plantas Medicinales }\end{array}$ & 44 & $\begin{array}{c}\text { Journal of } \\
\text { Venomous Animals } \\
\text { and Toxins }\end{array}$ & 2 & $\begin{array}{l}\text { Acta Botanica } \\
\text { Brasilica }\end{array}$ & 15 \\
\hline & $\begin{array}{l}\text { Brazilian Journal of } \\
\text { Plant Physiology }\end{array}$ & 37 & $\begin{array}{c}\text { Arquivo Brasileiro } \\
\text { de Medicina } \\
\text { Veterinária e } \\
\text { Zootecnia } \\
\end{array}$ & 1 & $\begin{array}{c}\text { Revista Brasileira } \\
\text { de Plantas } \\
\text { Medicinais }\end{array}$ & 7 \\
\hline & $\begin{array}{l}\text { Journal of soil science } \\
\text { and plant nutrition }\end{array}$ & 32 & Outras & 15 & Bragantia & 3 \\
\hline & $\begin{array}{c}\text { Revista Brasileira de } \\
\text { Plantas Medicinais }\end{array}$ & 30 & & & $\begin{array}{c}\text { Acta Scientiarum. } \\
\text { Agronomy }\end{array}$ & 2 \\
\hline & Biological Research & 22 & & & Acta Amazonica & 1 \\
\hline & Outras & 326 & & & Outras & 98 \\
\hline
\end{tabular}

$\mathrm{N}$ - número absoluto de publicações; EP1- expressão de pesquisa utilizando descritores em português; $\mathrm{EP} 2$ expressão de pesquisa utilizando descritores em inglês. $\mathrm{O}$ detalhamento das expressões consta na tabela 1. 
Como observado nesta pesquisa, o assunto plantas alelopáticas, independentemente da expressão de pesquisa, foi o que obteve o maior score com média de 93 publicações. Este aumento das pesquisas por plantas com propriedades alelopáticas deve-se principalmente a necessidade de entendimento sobre comunicação química que norteia esse processo e ocorre intra e inter espécies e, a possibilidade de aplicar esse conhecimento na agroecologia. Para tanto são realizados ensaios biológicos para avaliação da bioatividade de extratos, frações e compostos isolados de plantas, juntamente com técnicas de identificação e monitoramento dos aleloquímicos (NOLDIN et al. 2003). Esses compostos podem ser produzidos em qualquer órgão vegetal, porém em concentrações muito baixas e com características intrínsecas à planta, e são vistos como alternativas a agroquímicos sintéticos, visando o manejo sustentável e ecológico na produção agrícola (MARASCHIN-SILVA; ÁQUILA, 2006; DÍAZ-TIELAS et al., 2016).

Nas últimas décadas a comunidade científica tem despertado interesse por pesquisas com alelopatia, em diferentes partes do mundo. Uma tendência também observada no Brasil, demonstrado pelo número cada vez maior de artigo científico publicado em periódicos nacionais e internacionais (SOUZA FILHO, 2010).

No Brasil, investigações sobre o potencial alelopático das espécies nativas ainda é escasso, o que se torna necessário o conhecimento de aleloquímicos, pela variedade e riqueza da flora. Contudo, pesquisas com espécies nativas vem sendo realizadas como uma estratégia para adaptação defensiva ou ofensiva no estabelecimento de ervas daninhas (SILVA e AQUILA, 2006), como estratégia adaptativa, por exemplo, as espécies da caatinga (CASTRO; FABRICANTE e ARAUJO, 2017), e como alternativa a agroquímicos sintéticos para o manejo sustentável e ecológico da produção agrícola (ALVES et al., 2018; DIAS et al., 2005).

Destacou-se também a pesquisa por agentes antioxidantes de origem vegetal, com segundo maior score com média de 52 publicações. Esse interesse por plantas antioxidantes deve-se a necessidade de obtenção de agentes naturais para o combate aos radicais. Os radicais livres estão diretamente relacionados a várias patologias que acometem o homem, devido sua ação antiinflamatória, que são fundamentais para benefícios anticancerígenos, antienvelhecimento e de proteção contra doenças cardiovasculares, diabetes, obesidade e doenças neurodegenerativas (ZHANG et al., 2015). A possibilidade de obter nas plantas substâncias antiradicalares que possam atuar no combate ou controle destas fontes e/ou servirem como modelos para novos fármacos 
com esta ação, tem servido como estímulo para a pesquisa científica nesta área. Nos últimos anos, segundo Souza, Vieira e Putti (2018) houve um interesse crescente na determinação de fontes dietéticas adequadas de compostos fenólicos antioxidantes. Atualmente estudos envolvendo o potencial antioxidante em extratos e/ou substâncias presentes em frutos, sementes, folhas e raízes de plantas, tem cada vez mais frequente no uso de fisiopatologias.

Para a busca de artigos, quando se utilizou as expressões de pesquisa plantas AND antibacterianas ou plant AND antibacterial, a revista que mais publicou sobre o assunto foi a Revista Brasileira de Farmacognosia, com 2 e 3 publicações, respectivamente. Como já dito, este número baixo de publicações pode ser decorrente da inadequação da expressão de pesquisa utilizada, uma vez que o potencial antibacteriano de plantas tem sido alvo de muitas pesquisas nacionais e internacionais há vários anos (OLIVEIRA et al., 2006). A pesquisa por antibacterianos de origem vegetal possibilita alternativas para o uso de antibióticos caros e que podem gerar multirresistência a partir do uso frequente desses agentes terapêuticos. Segundo os pesquisadores Dahanukar; Kulkarni; Rege (2000), é imprescindível encontrar outras alternativas que possibilitem tratamentos eficazes de infecções bacterianas, como a utilização de extratos vegetais para a terapia antimicrobiana para combater doenças tem sido promissora desde os tempos antigos. $\mathrm{O}$ efeito sinérgico da associação de antibiótico com extratos de plantas contra bactérias resistentes pode levar a novas opções no tratamento de doenças infecciosas, quando o antibiótico não for mais eficaz por si só, durante o tratamento terapêutico (AIYEGORO; OKOH, 2009).

De um modo geral, um fato interessante chama atenção nesta pesquisa, dois periódicos brasileiros Planta Daninha e Revista Brasileira de Farmacognosia foram os que mais se destacaram em publicação de artigos (Tabela 2), o que mostra o potencial e a velocidades de propagação das informações científicas oriundas das nossas Universidades e dos Centros de Pesquisas, os quais envolvem alunos de graduação, mestrados e doutorados. Este fato também foi observado por Mendonça et al (2008)

Foi observado que para a busca pelos assuntos plantas antioxidante ou antimicrobianas, mesmo utilizando a expressão de pesquisa em português, a maioria das publicações são em idioma inglês. Enquanto que para a busca por plantas alelopáticas, tanto utilizando a expressão de pesquisa em português como em inglês, a diferença no quantitativo de publicações recuperadas nos idiomas inglês e português, é menor que 10\%. Certamente esta característica é decorrente da necessidade de internacionalização, 
que faz com que as revistas adotem em suas normas a obrigatoriedade de submissão dos trabalhos científicos em inglês ou também em inglês (Tabela 3).

Tabela 3. Característica das publicações recuperadas na busca na base de dados bibliográficos SciELO.

\begin{tabular}{|c|c|c|c|c|c|c|c|}
\hline \multicolumn{2}{|c|}{ CRITÉRIOS } & \multicolumn{2}{|c|}{$\begin{array}{c}\text { PLANTAS } \\
\begin{array}{c}\text { ANTIOXIDANTES } \\
(\%)\end{array} \\
\end{array}$} & \multicolumn{2}{|c|}{$\begin{array}{c}\text { PLANTAS } \\
\text { ANTIMICROBIANA } \\
\mathrm{N}(\%)\end{array}$} & \multicolumn{2}{|c|}{$\begin{array}{r}\text { PLANTAS } \\
\text { ALELOPÁTICAS } \\
(\%)\end{array}$} \\
\hline & & EP1 & $\mathrm{EP} 2$ & $\mathrm{EP} 1$ & $\mathrm{EP} 2$ & EP1 & $\mathrm{EP} 2$ \\
\hline \multirow{6}{*}{ IDIOMA } & & 72 & 355 & & 14 & 54 & 59 \\
\hline & Inglês & $(43,11)$ & $(63,51)$ & $2(40)$ & $(66,67)$ & $(40,30)$ & $(45,74)$ \\
\hline & & 69 & 131 & & 3 & & \\
\hline & Espanhol & $(41,32)$ & $(23,43)$ & - & $(14,29)$ & $7(5,22)$ & $9(6,98)$ \\
\hline & & 26 & 73 & & 4 & 73 & 61 \\
\hline & Português & $(15,57)$ & $(13,06)$ & $3(60)$ & $(19,05)$ & $(54,48)$ & $(47,29)$ \\
\hline \multirow{4}{*}{$\begin{array}{c}\text { TIPO DE } \\
\text { LITERATURA }\end{array}$} & Artigo de & 162 & 535 & 21 & 21 & 132 & 126 \\
\hline & pesquisa & $(97,59)$ & $(95,88)$ & $(100)$ & $(100)$ & $(98,51)$ & $(97,67)$ \\
\hline & Artigo de & & & & & & \\
\hline & revisão & $4(2,41)$ & $23(4,12)$ & - & - & $2(1,49)$ & $3(2,33)$ \\
\hline
\end{tabular}

O tipo de literatura mais utilizada foi artigo de pesquisa para todos os assuntos e suas expressões de pesquisa utilizadas na busca (Tabela 3). Os artigos de pesquisa normalmente são mais numerosos devido à necessidade de comprovação da eficácia das plantas como recurso medicinal ou fonte de compostos bioativos. $\mathrm{O}$ conhecimento que as pessoas têm sobre o potencial bioativo das plantas, desperta o interesse de pesquisadores, colaborando para o aumento deste tipo de pesquisas (BRITO; SENNAVALLE, 2011). Os artigos de revisão bibliográfica são uma forma de pesquisa que utilizam fontes de informações bibliográficas de outros autores para obtenção de resultados de pesquisa com o objetivo de fundamentar um determinado assunto (MESQUITA et al., 2006). A dificuldade em publicar artigos na categoria de revisão certamente impulsiona os pesquisadores a investir seus estudos na produção de artigos de pesquisa.

A primeira publicação ocorreu em 1983 para o assunto plantas alelopáticas e utilizando a expressão de pesquisa em português. O maior número de publicações foi registrado em 2010 (12 publicações) para a expressão de pesquisa em português, e em 
2013 (14 publicações) para a expressão em inglês. Para o assunto plantas antimicrobianas o primeiro registro ocorreu em 1999 (1 publicação) e não foi observado um aumento acentuado neste número, que como já foi dito, pode ser decorrente do uso das expressões de pesquisa inadequadas para o assunto na base SciELO. Com relação às plantas antioxidantes, a primeira publicação ocorreu em 1998 (1 publicação) para a expressão de pesquisa em inglês e em 2001 (1 publicação) para a expressão de pesquisa em português. A partir do ano de 2005 as publicações com plantas antioxidantes intensificaram-se, e 2015 destacou-se como o ano com a maior frequência de publicações, 68 publicações recuperadas pela expressão de pesquisa em inglês e 21 na expressão de pesquisa em português. De um modo geral foi possível observar que o assunto que mais chamou atenção dos pesquisadores neste período de tempo foi plantas antioxidante (Figura 2).

Figura 2. Produção científica por ano, sobre plantas antioxidantes, antimicrobianas e alelopáticas no período de 1983 a julho de 2018 na base de dados bibliográficos SciELO.

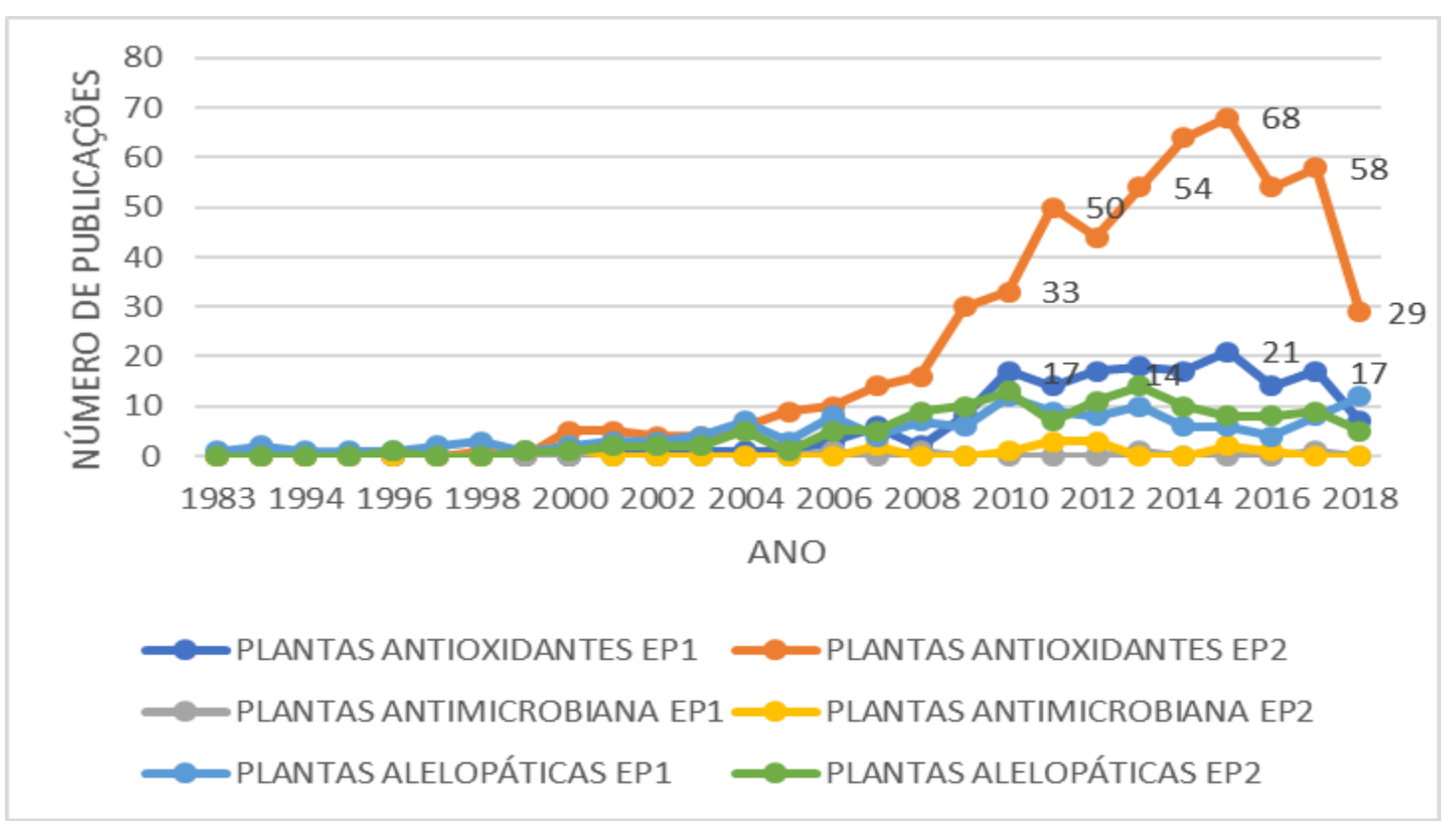

EP1- expressão de pesquisa utilizando descritores e português; EPQ expressão de pesquisa utilizando descritores em inglês. $\mathrm{O}$ detalhamento das expressões consta na tabela 1.

Este grande número de publicações serviu para divulgação de pesquisas etnobotânicas, que apresentaram um crescimento expressivo nas últimas décadas em muitas partes do mundo (CAVALCANTI; FAGUNDES; RAMACCIOTTI, 2005). Além 
disso, os antioxidantes naturais são de grande interesse em diversas áreas, incluindo alimentos (conservantes) e farmacologia (proteção do organismo contra o dano oxidativo) e, os produtos naturais vêm tendo, atualmente, ampla aplicação terapêutica com a difusão da ideia de que são mais seguros do que medicamentos sintéticos e, inclusive, são fontes naturais de substâncias antioxidantes (VELLOSA; BARBOSA; OLIVEIRA, 2007).

As áreas de publicações na base de dados Scielo com as temáticas plantas antioxidantes, antimicrobiana e alelopáticas são apresentadas na tabela 4. Computou-se 7 áreas de publicações: ciências agrárias, ciência da saúde, ciências biológicas, ciências exatas e da terra, ciências sociais aplicadas, engenharias e multidisciplinar. Foi observado o maior número de publicação nas áreas de ciências agrárias, ciências da saúde e ciências biológicas, para os assuntos plantas antioxidante e antimicrobiana. Já para o assunto plantas alelopáticas estudos tem sido desenvolvidos principalmente nas áreas ciências agrárias e ciências biológicas (Tabela 4).

Tabela 4. Relação das áreas com maior número de publicações nas temáticas plantas antioxidantes, antimicrobiana e alelopáticas em função da expressão de pesquisa.

\begin{tabular}{|c|c|c|c|c|c|c|}
\hline \multirow[t]{2}{*}{ ÁREAS } & \multicolumn{2}{|c|}{$\begin{array}{c}\text { PLANTAS } \\
\text { ANTIOXIDANTES } \\
\mathrm{N}(\%)\end{array}$} & \multicolumn{2}{|c|}{$\begin{array}{c}\text { PLANTAS } \\
\text { ANTIMICROBIANA } \\
\mathrm{N}(\%)\end{array}$} & \multicolumn{2}{|c|}{$\begin{array}{c}\text { PLANTAS } \\
\text { ALELOPÁTICAS } \\
\mathrm{N}(\%)\end{array}$} \\
\hline & $\mathrm{EP} 1$ & $\mathrm{EP} 2$ & $\mathrm{EP} 1$ & $\mathrm{EP} 2$ & EP1 & $\mathrm{EP} 2$ \\
\hline Ciências Agrárias & $66(31,58)$ & $184(27,92)$ & $1(20,00)$ & $5(17,85)$ & $118(86,13)$ & $85(61,15)$ \\
\hline Ciências da Saúde & $63(30,14)$ & $172(26,10)$ & $2(40,00)$ & $11(39,29)$ & $\mathrm{O}(0,00)$ & $6(4,32)$ \\
\hline Ciências Biológicas & $53(25,36)$ & $201(30,50)$ & $\mathcal{2}(40,00)$ & $9(32,14)$ & $15(10,95)$ & $40(28,78)$ \\
\hline $\begin{array}{l}\text { Ciências Exatas e da } \\
\text { Terra }\end{array}$ & $7(3,35)$ & $44(6,68)$ & - & - & $1(0,73)$ & $3(2,16)$ \\
\hline $\begin{array}{l}\text { Ciências Sociais } \\
\text { Aplicadas }\end{array}$ & $1(0,48)$ & $1(0,15)$ & - & - & - & - \\
\hline Engenharias & $8(3,83)$ & $15(2,28)$ & - & - & - & - \\
\hline Multidisciplinar & $11(5,26)$ & $42(6,37)$ & - & $3(10,71)$ & $3(2,19)$ & $5(3,60)$ \\
\hline
\end{tabular}

$\mathrm{N}$ - número absoluto de publicações; (\%) - percentual de publicações; EP1- expressão de pesquisa utilizando descritores em português; $\mathrm{EP} 2$ expressão de pesquisa utilizando descritores em inglês. O detalhamento das expressões consta na tabela 1. 
Essas características são decorrentes das especificidades de cada assunto e sua similaridade com as áreas temáticas. Esta relação norteia o pesquisador na escolha da revista, que por sua vez está cadastrada na área temática com maior adesão. Também foi observado que não existe um padrão de regularidade entre o número de publicações por área temática em função da expressão de pesquisa utilizada. Para cada área temática foi observado um quantitativo diferenciado em publicações recuperadas (Tabela 4).

Analisando o quantitativo de publicações na base de dados Web of Science (Wos), através de sua interface com a base Scielo, constatou-se um maior número de publicações para o assunto plantas antioxidantes, recuperados com a expressão de pesquisa em português, nas áreas temáticas de Farmacologia e farmácia (40), Ciências das plantas (29) e Agricultura multidisciplinar (20). Artigos recuperados com a expressão de pesquisa em inglês foram principalmente publicados nas as áreas Farmacologia e farmácia (1 11 ), Ciências das plantas (104) e Biologia (60). Áreas temáticas com o número reduzido de publicação (entre 1 a 5 ) foram classificadas como outros, apresentando 41 e 50 áreas diferentes, com 84 artigos em português e 116 em inglês publicados no total (Figura 3).

Figura 3. Publicações referentes a plantas antioxidantes por área temática Wos e expressão de pesquisa.

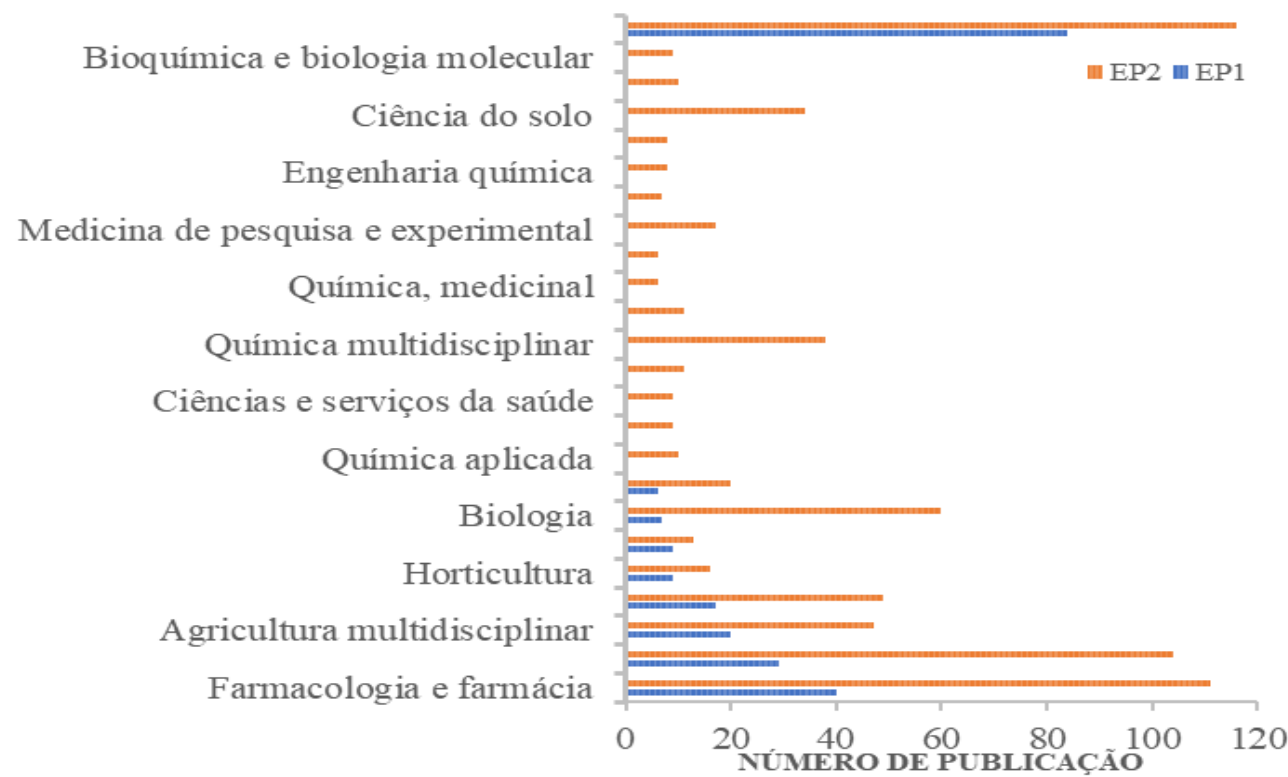

EP1- expressão de pesquisa utilizando descritores em português; EP2 expressão de pesquisa utilizando descritores em inglês. $\mathrm{O}$ detalhamento das expressões consta na tabela 1. 
Para o assunto plantas antimicrobianas (Figura 4), foram identificadas publicações em apenas 7 áreas na base Web of Science (Wos). Números maiores de publicações foram recuperados através do uso da expressão de pesquisa em inglês, com predominância nas áreas de Farmacologia e farmácia (5), Agricultura multidisciplinar (3) e Biologia (3).

Figura 4. Publicações referentes a plantas antimicrobianas por área temática Wos e expressão de pesquisa.

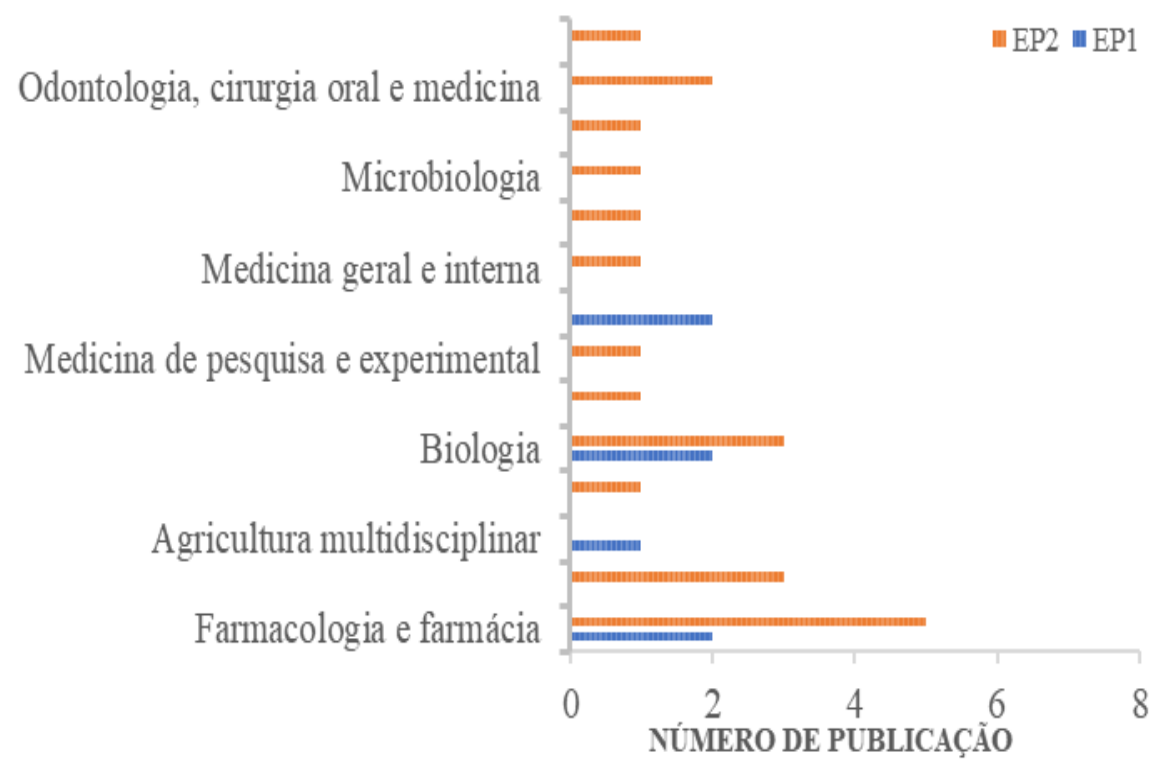

EP1- expressão de pesquisa utilizando descritores em português; EP2 expressão de pesquisa utilizando descritores em inglês. $\mathrm{O}$ detalhamento das expressões consta na tabela 1 .

Estudos com plantas com efeitos alelopáticos vem sendo publicados na base de dados Wos (Figura 5) principalmente na expressão de pesquisa em inglês, com maior número de publicações na área de Agricultura multidisciplinar (71 publicações). Essa concentração na área deve-se a potencial aplicação dos produtos da pesquisa na agroecologia ou manejo alternativo de plantas. 
Figura 5. Publicações referentes a plantas alelopáticas por área temática Wos e

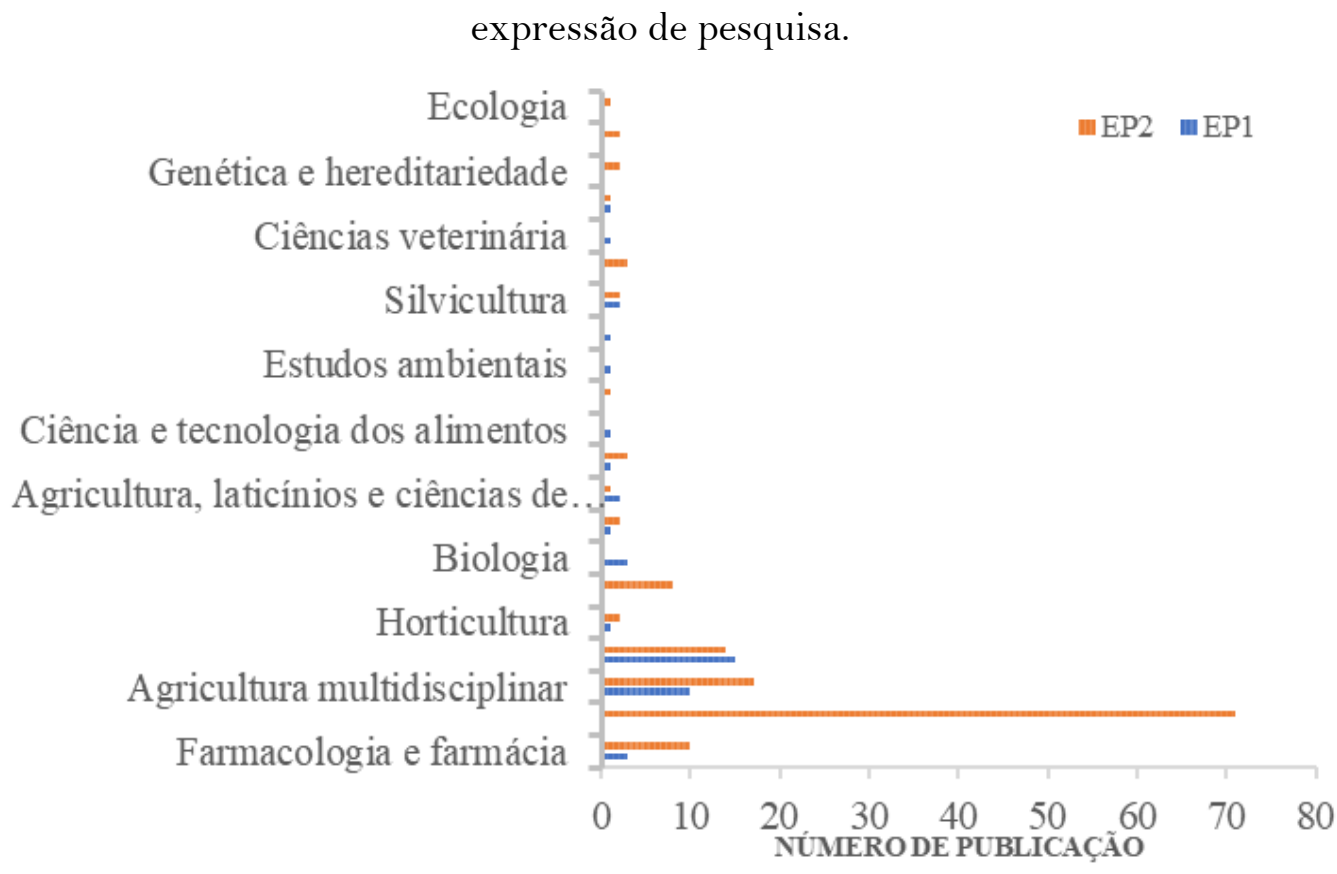

EP1- expressão de pesquisa utilizando descritores em português; EPQ expressão de pesquisa utilizando descritores em inglês. $\mathrm{O}$ detalhamento das expressões consta na tabela 1.

\section{CONSIDERAÇÕES FINAIS}

As plantas, ao longo do tempo, têm sido utilizadas pela população para satisfazer suas mais diversas necessidades. Seu uso tem se destacado na comunidade científica em decorrência de seu potencial de suas propriedades terapêuticas, como antioxidantes e antimicrobianas, como também por ser fonte de aleloquímicos, podendo ser úteis também na agroecologia. Pesquisas voltadas para o estudo e avaliação deste potencial biológico das espécies vegetais tem de ser estimulados a descoberta de novas biomoléculas e novos agentes que possam ser utilizados em produtos farmacêuticos, cosméticos e na indústria agroindústria, que pode ser acompanhada e analisada através da base de dados bibliográfica Scielo. Foi observado que a pesquisa com plantas antioxidantes e alelopáticas, apresentam um quantitativo maior que com plantas antimicrobianas, nesta base de dados, o que ressalta a importância de usar diferentes expressões de pesquisa e mais de uma base de dados bibliográfica, durante uma pesquisa exploratória. Através desta análise cenciométrica também foi possível verificar o 
interesse da ciência e de pesquisadores sobre o potencial antioxidante e alelopático das plantas, e a tendência de produção científica nessa área.

\section{REFERÊNCIAS}

1. ALVES, R. M. et al. Potencial alelopático de folhas secas de Caesalpinia férrea Mart. Em diferentes períodos de decomposição sobre a germinação de Vigna unguiculata (L.) Walp, cv. Canapu. Enciclopédia Biosfera, v.15, n.27, p.200-207, 2018.

2. BONA, E. A. M. et al. Comparação de métodos para avaliação da atividade antimicrobiana e determinação da concentração inibitória mínima (cim) de extratos vegetais aquosos e etanólicos. Arquivos do Instituto Biológico, v.81, n.3, p. $218-225,2014$.

3. BRASIL. Ministério da Saúde. Plantas de Interesse ao SUS. Brasília, 2013. Disponível em: . Acesso em: agosto de 2018.

4. BRITO, M. R.; SENNA-VALLE, L. Plantas medicinais utilizadas na comunidade caiçara da Praia do Sono, Paraty, Rio de Janeiro, Brasil. Acta Botânica Brasilica, v. 25, p. 363-372, 2011.

5. CAstro, R. A.; FAbrichnte, J. R.; ARAujO, K. C. T. Sociabilidade e potencial alelopático de espécies da caatinga sobre a invasora Nicotiana glauca Graham (Solanaceae). Natureza online, v.15, n.1, p.59-69, 2017.

6. CAVAlCANTI, L. R. M. T.; FAGUNDES, M. E. M.; RAMACCIOTTI R. E. L. Distribuição regional dos fluxos de recursos federais para a ciência e tecnologia. Revista Parcerias Estratégicas, v. 10, p. 59-78, 2005.

7. DAhanUKAR, S. A.; KULKARni, R. A.; REGE, N. N. Pharmacology of medicinal plants and natural products. The Indian Journal of Pharmacology, v. 32 , p. 81-118, 2000 .

8. DIAS, G. M. et al. Contribuição ao estudo alelopático de Maytenus ilicifolia Mart. Ex Reiss, Celastraceae. Brazilian Journal of Pharmacognosy, v.15, n. 3, p. 220-223, 2005.

9. DÍAZ-TIELAS, C.; GRAÑA, E.; REIGOSA, M. J.; SÁNCHEZ-MOREIRAS, A. M. Biological activities and novel applications of chalcones. Planta Daninha, v. 34, n. 3, p. 607-616, 2016. 
10. DROGE, W. Free radicals in the physiological control of cell function. Physiological Reviews, n. 82, p.47-95, 2002.

11. GONÇALVEZ, A. L.; ALVES FILHO A.; MENEZES H. Estudo comparativo da atividade antimicrobiana de extratos de algumas árvores nativas. Arquivo Instituto Biológico v.72, n.3, p.353-358, 2005.

12. MARASCHIN-SILVA, F.; AQUILA, M.E.A. Potencial alelopático de espécies nativas na germinação e crescimento inicial de Lactuca sativa L. (Asteraceae). Acta Botânica Brasílica, v. 20, p.61-69, 2006.

13. MARMitT, D. J.; REMPEL, C.; GIETTERT, M. I.; Silva, A. C. Análise sistemática da produção científica do zingiber officinale Roscoe após a criação da relação nacional de plantas medicinais de interesse ao sistema único de saúde. Arquivos de Ciências da Saúde, v. 22, n. 4, p. 14-2 1, 2015.

14. MEGRAJ, K. V. K.; et al. Biological activities of some Indian medicinal plants. Journal of Advanced Pharmacy Education \& Research, v.1, p.12-44, 2011.

15. MENDONÇA, R. C.; FELFILI, J. M.; WALTER, B. M. T.; SILVA-JUNIOR, M. C.; REZENDE, A. V.; FIlgueiras, T. S.; NOGUEIRA, P. E. E.; FAGG, C. W. Flora Vascular do Cerrado: checklist com 12.356 espécies. In: ALMEIDA, S. M.; SANO, S. P.; RIBEIRO, J. F. (Ed.). Cerrado: Ecologia e Flora. Brasília: Embrapa Informação Tecnológica, v. 2, p. 422-442, 2008.

16. MENDONÇA, R. J.; FELFILI, B.; WALTER, J.C.; SILVA JR.; REZENDE, A.; FILGUEIRAS, T.; NOGUEIRA, P. Flora vascular do Cerrado. In: SANO, S.; ALMEIDA, S. (eds.). Cerrado. Ambiente e flora. p. 288-556. Empresa Brasileira de Pesquisa Agropecuária - Embrapa - Cerrados, Planaltina, Brasil. 1998.

17. MESQUITA, R; BRAMBILLA, S.; LAIPELT, R. C; MAIA, M. F.; VANZ, S.; CAREGNATO, S. E. Elaboração e aplicação de instrumentos para avaliação da base de dados Scopus. Perspectiva em Ciência da Informação, v. 11, p. 187-205, 2006.

18. MORAIS, S. M.; CATUNDA-JR, E. A.; SIlVA, A. R. A, MARTINS-NETO, J. S. Atividade antioxidante de óleos essenciais de espécies de Croton do Nordeste do Brasil. Química Nova, v.29, p. 907-910, 2006.

19. MORENO, A. H. et al. Avaliação da atividade antimicrobiana e citotoxicidade hemolítica em diferentes extratos vegetais. Arquivos de Ciência e Saúde, v.25, n.1, p.11-12, 2018. 
20. NOLDIN, V.F. et al. Composição química e atividades biológicas das folhas de Cynara scolymus L. (Alcachofra) cultivada no Brasil.Química Nova, v. 26, p. 331334, 2003.

21. OlIVEIRA, R. A. G.; LIMA, E. O.; VIEIRA, W. L.; FREIRE, K. R. L.; TRAJANO, V. N.; LIMA, I. O.; SOUZA, E. L.; TOLEDO, M. S.; SILVAFILHO, R. N. Estudo da interferência de óleos essenciais sobre a atividade de alguns antibióticos usados na clínica. Revista Brasileira de Farmacognosia, v. 16, n. 1, p. 77-82, 2006.

22. PACKER, A.. L.; MENEGHINI, R. O SciELO aos 15 anos: raison d'être, avanços e desafios para o futuro. In: E. SciELO - 15 Anos de Acesso Aberto [livro eletrônico: um estudo analítico sobre Acesso Aberto e comunicação científica. Packer, A. L, Cop, N., Luccisano, A., Ramalho, A. e Spinak (Org.). Paris: UNESCO, 2014.

23. PIÑA-RODRIGUES, F. C. M.; LOPES, B. M. Potencial alelopático de Mimosa caesalpinaefolia Benth sobre sementes de Tabebuia alba (Cham.) Sandw. Floresta e Ambiente. V. 8, n.1, p.130 - 136, 2001.

24. PIOVESAN, A.; TEMPORINI, E. R. Pesquisa exploratória: procedimento metodológico para o estudo de fatores humanos no campo da saúde pública. Rev. Saúde Pública, v. 29, n. 4, p. 318-325, 2015.

25. RIBEIRO, C. M. et al. Avaliação da atividade antimicrobiana de plantas utilizadas na medicina popular da Amazônia. Infarma, v.21, n.1, p.45-49, 2009.

26. SILVA, F. M.; AQUILA, M. E. A. Potencial alelopático de espécies nativas na germinação e crescimento inicial de Lactuca sativa L. (Asteraceae). Acta Botânica Brasílica, v.20, n.1, p. 61-69, 2006.

27. SOUZA FILHO, A. P. da S. et al. Efeitos potencialmente alelopáticos dos óleos essenciais de Piper hispidinervium C. DC. e Pogostemon heyneanus Benth sobre plantas daninhas. Acta amazônica. vol. 39. n. 2. p. 389- 396. 2009.

28. SOUZA FILHO, A. P.S.; GUILHON, G.M.S.P.; SANTOS, L.S. Metodologias empregadas em estudos de avaliação da atividade alelopática em condições de laboratório: revisão crítica. Planta daninha, v. 28, n. 3, p. 689-697, 2010.

29. SOUZA, A. V.; VIEIRA, M. R. S.; PUTTI, F. F. Correlações entre compostos fenólicos e atividade antioxidante em casca e polpa de variedades de uva de mesa. Brazilian Journal of Food Technology, v. 21, p.1-6, 2018. 
30. SOUZA, L.S. et al. Efeito alelopático de capim-braquiária (Brachiaria decumbens) sobre o crescimento inicial de sete espécies de plantas cultivadas. Planta Daninha. Sociedade Brasileira da Ciência das Plantas Daninhas, v. 24, n. 4, p. 657-668, 2006. Disponível em: <http://hdl.handle.net/11449/5751>.

31. VARJABEDIAN, R. Lei da Mata Atlântica: retrocesso ambiental. Estudos avançados, v. 24, n.68, 2010.

32. VEllosA. J. C. R.; BARbOSA V. F.; OliveiRA, O. M. M. F. Pesquisa de produtos naturais: plantas e radicais livres Revista Eletrônica de Farmácia, v. IV, n. 2, p. 119-130, 2007.

33. VENDRUSCOLO, G.S. \& MENTZ, L.A. 2006. Estudo da concordância das citações de uso e importância das espécies e famílias utilizadas como medicinais pela comunidade do bairro Ponta Grossa, Porto Alegre, RS, Brasil. Acta Botânica Brasilica 20: 367-382.

34. ZHANG, YJ et al. Antioxidant phytochemicals for the prevention and treatment of chronic disease. Molecules, v. 20, p. $21138-56,2015$. 